\title{
Análise crítica das opções terapêuticas para deformidades do sulco lacrimal: revisão da literatura
}

\section{Critical analysis of options for tear trough: literature review}

William de Almeida Machado ${ }^{1}$. Salustiano Gomes de Pinho Pessoa². Vitor Vasconcellos Muniz ${ }^{3}$.

1 Membro Aspirante da Sociedade Brasileira de Cirurgia Plástica - SBCP, médico residente do Serviço de Cirurgia Plástica e Microcirurgia Reconstrutiva do Hospital Universitário Walter Cantídio (HUWC), Universidade Federal do Ceará (UFC), Fortaleza, Ceará, Brasil. 2 Membro Titular da Sociedade Brasileira de Cirurgia Plástica (SBCP), MBA em Gestão de Organizações Hospitalares e Sistemas de Saúde, Fundação Getúlio Vargas, Mestrado em Cirurgia, Universidade Federal do Ceará (UFC), Fortaleza, Ceará, Brasil. 3 Membro Titular da Sociedade Brasileira de Cirurgia Plástica (SBCP), Pós-graduação em cirurgia plástica pela Universidade Federal do Ceará (UFC), Preceptor do Serviço de Cirurgia Plástica e Microcirurgia Reconstrutiva da UFC, Fortaleza, Ceará, Brasil.

\section{RESUMO}

O sulco nasojugal, também conhecido como canal lacrimal, é um sulco cutâneo distinto que se estende inferolateralmente do canto medial até, aproximadamente, a linha pupilar média e se torna progressivamente mais proeminente com a idade. O contorno anormal de tecidos periorbitais resultam em uma área escura que denota a aparência cansada. A concavidade criada por esse sulco é comumente percebida como um círculo escuro abaixo do olho que é difícil de esconder com maquiagem. A atrofia óssea e gordurosa com a idade pode contribuir com a perda do suporte tecidual e descida da bochecha, que aprofunda o sulco lacrimal. O trabalho em questão tem como objetivo a revisão de literatura referente ao tratamento da deformidade do sulco lacrimal.

Palavras-chave: Cirurgia plástica. Revisão. Face. Olho. Envelhecimento.

\section{ABSTRACT}

The nasojugal groove, also known as tear duct is a distinct skin groove extending inferolaterally from the medial canthus to approximately the middle pupillary line and becomes progressively more prominent with age. Abnormal contour of periorbital tissues result in a dark area denotes the tired appearance. The concavity created by this groove is commonly perceived as a dark circle under the eye that is hard to hide with makeup. Bone and fat atrophy with age may contribute to the loss of tissue support and also the lowering of the cheek, which deepens the lacrimal groove. The work in question aims to literature review related to the treatment of tear trough.

Keywords: Surgery, plastic. Review. Face. Eye. Aging.

Autor correspondente: William de Almeida Machado, Rua da paz 455, apartamento 602, Fortaleza, Ceará. CEP: 60165-180. Telefone: +55 85 988040983. E-mail: will.machado@gmail.com

Conflito de interesses: Não há qualquer conflito de interesses por parte de qualquer um dos autores.

Recebido em: 09 Abr 2016; Revisado em: 08 Mai 2016; Aceito em: 10 Mai 2016. 


\section{INTRODUÇÃO}

O sulco nasojugal, também conhecido como canal lacrimal, é um sulco cutâneo distinto que se estende inferolateralmente do canto medial até, aproximadamente, a linha pupilar média e se torna progressivamente mais proeminente com a idade. O contorno anormal de tecidos periorbitais resultam em uma área escura que denota a aparência cansada. ${ }^{1}$ A concavidade criada por esse sulco é comumente percebida como um círculo escuro abaixo do olho que é difícil de esconder com maquiagem. A atrofia óssea e gordurosa com a idade pode contribuir com a perda do suporte tecidual e descida da bochecha, que aprofunda o sulco lacrimal. ${ }^{1}$

Foi descrito pela primeira vez em 1961 por Duke-Elder e Wybar e nomeado como "deformidade do sulco lacrimal" em 1969 por Flowers. $^{2}$ Neste ano, Loeb e Flowers publicaram técnicas cirúrgicas destinadas a correção da deformidade do sulco lacrimal. ${ }^{3}$ Desde então, várias técnicas têm sido descritas para correção dessa deformidade.

\section{OBJETIVO}

O presente trabalho tem como objetivo a revisão da literatura pertinente ao tratamento da deformidade do sulco lacrimal, com análise descritiva e crítica dos métodos terapêuticos disponíveis.

\section{MÉTODO}

O estudo consiste em uma revisão sistemática da literatura especializada. Foram selecionados artigos publicados nas bases de dados Scopus, PubMed, ScienceDirect e OneFile nos idiomas português e inglês a partir dos termos: "tear trough deformity" e "tratamento sulco nasojugal". Foram utilizados os seguintes critérios para seleção dos artigos: ensaios clínicos, meta-análises e artigos de revisão que abordassem majoritariamente o tema em questão.

\section{RESULTADOS}

Foram encontrados 556 artigos entre o ano de 1990 e 2015, sendo selecionadas, para análise, 37 publicações englobando a abordagem clínico-cirúrgica da afecção em questão. Os trabalhos foram avaliados de acordo com os métodos terapêuticos utilizados pelos autores (Quadro 1).

Dos trabalhos analisados, 43\% (16 trabalhos) enfatizavam o reposicionamento de gordura como tratamento para a deformidade do sulco lacrimal, enquanto em 13 publicações o preenchimento com ácido hialurônico foi o tratamento em estudo. Foram descritos ainda tratamentos como lipoenxertia (2 trabalhos), implante de silicone (2 trabalhos), enxerto dérmico acelular (1 trabalho), técnica da polia periostal (1 trabalho), suspensão facial com fios de sutura (1 trabalho) e cobertura com músculo orbicular (1 trabalho).

A data de publicação dos trabalhos variou de uma publicação nos anos de 1993, 1995 e 2009, duas publicações nos anos de 1999, 2007, 2008, 2011, 2012 e 2015, três publicações em 2005 e 2006, cinco publicações em 2010 e seis publicações em 2013 e 2014. (Quadro 1).

Quadro 01. Principais publicações sobre tratamento da deformidade do sulco nasojugal entre 1990 e 2015.

\begin{tabular}{|l|l|l|l|l|l|}
\hline & TRABALHO & AUTOR & N* & ANO & TIPO DE TRATAMENTO \\
\hline 1 & Tear Trough Implants for Correction of Tear Trough Deformity & Flowers RS & - & 1993 & Implante de silicone \\
\hline 2 & $\begin{array}{l}\text { Arcus marginalis release and orbital fat preservation in midface } \\
\text { rejuvenation }\end{array}$ & Hamra & 1995 & Reposicionamento de gordura \\
\hline 3 & $\begin{array}{l}\text { The superficial subciliary cheek lift: A technique for } \\
\text { rejuvenating the infraorbital region and nasojugal groove: A } \\
\text { review of 71 patients }\end{array}$ & Moelleken B & - & 1999 & Reposicionamento de gordura \\
\hline 4 & $\begin{array}{l}\text { Transconjunctival Orbital Fat Repositioning: Transposition of } \\
\text { Orbital Fat Pedicles into a Subperiosteal Pocket }\end{array}$ & $\begin{array}{l}\text { Robert Alan Goldberg, } \\
\text { M.D. }\end{array}$ & 24 & 1999 & Reposicionamento de gordura \\
\hline 5 & $\begin{array}{l}\text { Lower Blepharoplasty: Transconjunctival Fat Repositioning } \\
\text { Barbed polypropylene sutures for mid-face elevation: Early } \\
\text { results }\end{array}$ & Lee S, Isse N & - & 2005 & Fios de sutura \\
\hline 7 & $\begin{array}{l}\text { Treatment of Tear Trough Deformity and Lower Lid Bowing } \\
\text { with Injectable Hyaluronic Acid }\end{array}$ & $\begin{array}{l}\text { Michael A. C. Kane } \\
\text { M.D. }\end{array}$ & 24 & 2005 & $\begin{array}{l}\text { Preenchimento com ácido } \\
\text { hialurônico }\end{array}$ \\
\hline 8 & $\begin{array}{l}\text { Correcting Suborbital Malar Hypoplasia and Related Boney } \\
\text { Deficiencies }\end{array}$ & Robert S. Flowers MD & 100 & 2005 & Reposicionamento de gordura \\
\hline 9 & $\begin{array}{l}\text { Cosmetic Lower Eyelid Blepharoplasty With Fat Repositioning } \\
\text { via Intra-SOOF Dissection: Surgical Technique and Initial } \\
\text { Outcomes }\end{array}$ & $\begin{array}{l}\text { Yasaman Mohadjer, } \\
\text { M.D et al }\end{array}$ & $\begin{array}{l}\text { Implante de silicone } \\
\text { Deep-Fill Hyaluronic Acid for the Temporary Treatment of the } \\
\text { Naso-Jugal Groove: A Report of 303 Consecutive Treatments }\end{array}$ & $\begin{array}{l}\text { Kenneth D. Steinsapir } \\
\text { and Techniques }\end{array}$ & 164 \\
\hline 11 & $\begin{array}{l}\text { Autologous Fat Transfer: an In-Depth Look at Varying Concepts } \\
\text { Kimberly J. Butterwick, } \\
\text { MD }\end{array}$ & $\begin{array}{l}\text { Preenchimento com ácido } \\
\text { hialurônico }\end{array}$ \\
\hline
\end{tabular}

Continua. 
Conclusão.

Quadro 01. Principais publicações sobre tratamento da deformidade do sulco nasojugal entre 1990 e 2015.

\begin{tabular}{|c|c|c|c|c|c|}
\hline & TRABALHO & AUTOR & $\mathrm{N}^{*}$ & ANO & TIPO DE TRATAMENTO \\
\hline 12 & $\begin{array}{l}\text { Hyaluronic Acid Injections for Correction of the Tear Trough } \\
\text { Deformity }\end{array}$ & Val S. Lambros, M.D. & 200 & 2007 & $\begin{array}{l}\text { Preenchimento com ácido } \\
\text { hialurônico }\end{array}$ \\
\hline 13 & $\begin{array}{l}\text { Transconjunctival Orbital Fat Repositioning for Tear Trough } \\
\text { Deformity in Young Asians }\end{array}$ & Akira Momosawa et al & 20 & 2008 & Reposicionamento de gordura \\
\hline 14 & $\begin{array}{l}\text { Blending of the eyelid-cheek junction and removal of protruding } \\
\text { fat: an intraoral approach to blepharoplasty of the lower eyelid }\end{array}$ & Haizhong Zhang et al & 17 & 2009 & $\begin{array}{l}\text { Reposicionamento de gordura } \\
\text { via intraoral }\end{array}$ \\
\hline 15 & An Integrated Approach to Lower Blepharoplasty & David A. Hidalgo, M.D. & 248 & 2010 & Reposicionamento de gordura \\
\hline 16 & Autologous Fat and Fillers in Periocular Rejuvenation & $\begin{array}{l}\text { Edward D. } \\
\text { Buckingham, MD }\end{array}$ & & 2010 & $\begin{array}{l}\text { Preenchimento com ácido } \\
\text { hialurônico }\end{array}$ \\
\hline 17 & $\begin{array}{l}\text { Periocular Rejuvenation: Lower Eyelid Blepharoplasty with Fat } \\
\text { Repositioning and the Suborbicularis Oculi Fat }\end{array}$ & Jonathan R. Grant et al & - & 2010 & Reposicionamento de gordura \\
\hline 18 & Periorbital rejuvenation surgery in the geriatric population & $\begin{array}{l}\text { Ann Yi-Chiun Chuang } \\
\text { et al }\end{array}$ & - & 2010 & Reposicionamento de gordura \\
\hline 19 & $\begin{array}{l}\text { Quantitative Evaluation of Volume Augmentation in the Tear } \\
\text { Trough with a Hyaluronic Acid-Based Filler: A 3D Analysis }\end{array}$ & $\begin{array}{l}\text { Alexander S. Donath } \\
\text { et al }\end{array}$ & 10 & 2010 & $\begin{array}{l}\text { Preenchimento com ácido } \\
\text { hialurônico }\end{array}$ \\
\hline 20 & $\begin{array}{l}\text { Use of Hyaluronic Acid Filler for Tear-Trough Rejuvenation as } \\
\text { an Alternative to Lower Eyelid Surgery }\end{array}$ & Ana M. S. Morley et al & 100 & 2010 & $\begin{array}{l}\text { Preenchimento com ácido } \\
\text { hialurônico }\end{array}$ \\
\hline 21 & $\begin{array}{l}\text { A novel technique for repositioning lower eyelid fat via the } \\
\text { transoral approach in association with midface lift }\end{array}$ & M. Mark Mofid & & 2011 & Reposicionamento de gordura \\
\hline 22 & $\begin{array}{l}\text { Fat repositioning via supraperiosteal dissection with internal } \\
\text { fixation for tear trough deformity in an Asian population }\end{array}$ & Shu Lang Liao et al & 212 & 2011 & Reposicionamento de gordura \\
\hline 23 & $\begin{array}{l}\text { Dermal Fillers for the Treatment of Tear Trough Deformity: A } \\
\text { Review of Anatomy, Treatment Techniques, and their Outcomes }\end{array}$ & Jaishree Sharad & & 2012 & $\begin{array}{l}\text { Preenchimento com ácido } \\
\text { hialurônico }\end{array}$ \\
\hline 24 & $\begin{array}{l}\text { Lipoenxertia autóloga periorbitária no rejuvenescimento facial: } \\
\text { análise retrospectiva da e cácia e da segurança em } 31 \text { casos }\end{array}$ & Chang Yung Chia et al & 32 & 2012 & Lipoenxertia \\
\hline 25 & $\begin{array}{l}\text { Acellular Dermal Grafts for Tear Trough Deformity in Revision } \\
\text { Lower Blepharoplasty }\end{array}$ & $\begin{array}{l}\text { Russell W. H. Kridel, } \\
\text { MD }\end{array}$ & & 2013 & Enxerto dérmico acelular \\
\hline 26 & $\begin{array}{l}\text { Hyaluronic Acid Filler Injections for Tear-Trough Deformity: } \\
\text { Injection Technique and High-Freq Ultrasound Follow-up } \\
\text { Evaluation }\end{array}$ & $\begin{array}{l}\text { Antonino De Pasquale } \\
\text { et al }\end{array}$ & 22 & 2013 & $\begin{array}{l}\text { Preenchimento com ácido } \\
\text { hialurônico }\end{array}$ \\
\hline 27 & $\begin{array}{l}\text { Rejuvenation effects of hyaluronic acid injection on nasojugal } \\
\text { groove: Prospective randomized split face clinical controlled } \\
\text { study }\end{array}$ & HEE-KYEONG LIM & 10 & 2013 & $\begin{array}{l}\text { Preenchimento com ácido } \\
\text { hialurônico }\end{array}$ \\
\hline 28 & $\begin{array}{l}\text { Semipermanent Volumization by an Absorbable Filler: Onlay } \\
\text { Injection Technique to the Bone }\end{array}$ & Takanobu Mashiko et al & 38 & 2013 & $\begin{array}{l}\text { Preenchimento com ácido } \\
\text { hialurônico }\end{array}$ \\
\hline 29 & $\begin{array}{l}\text { Volumetric Rejuvenation of the Tear Trough With Repo and } \\
\text { Ristow }\end{array}$ & Adi Einan-Lifshitz et al & 57 & 2013 & Reposicionamento de gordura \\
\hline 30 & $\begin{array}{l}\text { Calcium Hydroxyl-Apatite (Radiesse) for the Correction of } \\
\text { Periorbital Hollows, Dark Circles, and Lower Eyelid }\end{array}$ & $\begin{array}{l}\text { Francesco P. } \\
\text { Bernardini, M.D et al }\end{array}$ & 63 & 2014 & $\begin{array}{l}\text { Preenc c cálcio hidroxi- } \\
\text { apatita (radiesse) }\end{array}$ \\
\hline 31 & $\begin{array}{l}\text { Lower Lid Blepharoplasty Panel Discussion, Controversies, } \\
\text { and Techniques }\end{array}$ & Shan Baker et al & - & 2014 & Reposicionamento de gordura \\
\hline 32 & $\begin{array}{l}\text { Redraping of the fat and eye lift for the correction of the tear } \\
\text { trough }\end{array}$ & Ioannis E. Liapakis et al & 35 & 2014 & Reposicionamento de gordura \\
\hline 33 & $\begin{array}{l}\text { The periosteal pulley technique for orbital fat realignment and } \\
\text { correction of nasojugal groove in lower lid blepharoplasty }\end{array}$ & Kamol Wattanakrai et al & 115 & 2014 & Técnica da polia periostal \\
\hline 34 & $\begin{array}{l}\text { Transconjunctival Subperiosteal Fat Reposition for Tear Trough } \\
\text { Deformity }\end{array}$ & Seungki Youn et al & 120 & 2014 & Reposicionamento de gordura \\
\hline 35 & $\begin{array}{l}\text { Use of a Novel Orbicularis Oculi Muscle Overlap Method for } \\
\text { Correction of Tear-Trough Deformity }\end{array}$ & Yuzo Komuro et al & 10 & 2014 & $\begin{array}{l}\text { Cobertura com músculo } \\
\text { orbicular }\end{array}$ \\
\hline 36 & $\begin{array}{l}\text { Correction of tear trough deformity with a cohesive polydensi } \\
\text { ed matrix hyaluronic acid: a case series }\end{array}$ & $\begin{array}{l}\text { Jürgen Huber- } \\
\text { Vorländer }\end{array}$ & 6 & 2015 & $\begin{array}{l}\text { Preenchimento com ácido } \\
\text { hialurônico }\end{array}$ \\
\hline 37 & $\begin{array}{l}\text { Minimally invasive eyelid care in dermatology: Medical, laser, } \\
\text { and cosmetic therapies }\end{array}$ & Ramin Fathi et al & 244 & 2015 & $\begin{array}{l}\text { Preenchimento com ácido } \\
\text { hialurônico }\end{array}$ \\
\hline
\end{tabular}




\section{DISCUSSÃO}

O contorno anormal dos tecidos periorbitais resultam em uma área de depressão que denotam aparência triste. A sombra criada por essa depressão é comumente percebida como círculos escuros abaixo dos olhos que são difíceis de esconder com a maquiagem. Várias estruturas estão envolvidas na formação do sulco do canal lacrimal como o ligamento órbito-malar, bolsas de gordura palpebral inferior e malar. Com o envelhecimento ocorre uma atenuação do ligamento órbito-malar e descida da gordura malar expondo a gordura orbital e o sulco entre a superfície criada pelo contorno da gordura palpebral inferior e a borda da gordura malar. ${ }^{4}$ Este é conhecido como sulco nasojugal, junção pálpebro-malar ${ }^{2}$ ou, mais comumente, deformidade do sulco lacrimal. ${ }^{5}$

Várias técnicas para correção do sulco têm sido descritas. Alguns procedimentos mais complexos envolvem a elevação de tecidos desde a base da maxila, enquanto outros realizam a elevação do terço médio da face durante a ritidoplastia. ${ }^{6}$ Sasaki, em 2002, descreveu a utilização de fios de sutura subcutâneos na bochecha para promover a suspensão de terço médio da face. Tinha como vantagens o aumento volumétrico da parte superior da bochecha, preenchimento da deformidade do canal lacrimal principalmente em sua porção lateral e elevação da boca sobre o esqueleto dentário. ${ }^{6}$

Seguindo tendências cirúrgicas menos invasivas, alguns procedimentos simplesmente elevam partes moles da bochecha através de suturas de fixação através da incisão na pálpebra inferior. ${ }^{7}$ Esses procedimentos visam a correção do defeito através da suspensão de massas da borda inferior do mesmo (situadas na bochecha). Moelleken ${ }^{8}$ descreveu uma técnica de rejuvenescimento da região infraorbital e correção do sulco nasojugal por incisão subciliar estendendo-se a cerca de 4 a $10 \mathrm{~mm}$ do canto lateral. Uma faixa de músculo orbicular pré-tarsal $(4 \mathrm{~mm})$ é deixado intacto para manter o suporte da pálpebra inferior. Disseca-se entre as fibras do músculo orbicular, retira-se gordura periorbital tão somente haja herniação grosseira. Prossegue-se a dissecção inferiormente ao músculo orbicular e gordura malar, tipicamente por $4 \mathrm{~cm}$ inferior e $2 \mathrm{~cm}$ de lateral para medial ao canto lateral. Ressecase conservadoramente o excesso de músculo e pele. Faz-se a fixação do músculo orbicular e gordura malar à fáscia temporal intermediária. Realiza-se fechamento do músculo orbicular em um plano separado e fechamento da pele com aproximação exata da incisão lateral. ${ }^{8}$

Outros procedimentos atuam na tentativa de diminuir a borda superior do sulco (bolsas da pálpebra inferior) adicionando tecido para a área deficiente entre as duas bordas. Hamra ${ }^{9}$ popularizou o preenchimento do canal lacrimal com retalho de gordura vascularizada. O procedimento por ele descrito em 1992, acessava diretamente a borda superior do sulco e utilizava a gordura em aparente excesso deste lado para preencher a depressão através do reposicionamento da mesma soltando o arco marginal.

Em busca de técnicas menos invasivas, cirurgiões tem descrito transposição de gordura infraorbital via acesso transconjuntival com fixação externa inicialmente e depois com pontos de fixação interna. ${ }^{10}$ Golberg, ${ }^{11}$ em 1999, publicou um trabalho demonstrando a correção da deformidade do sulco através da reposição da gordura orbital pela incisão transconjuntival. Esse acesso é realizado da carúncula até o fórnix lateral, expõe-se a borda orbital ao nível do arco marginal usando dissecção romba, faz-se uma bolsa subperiostal normalmente estendendo-se para a face da maxila até $1,5 \mathrm{~cm}$ do forame infraorbital. As bolsas de gordura medial e central são dissecadas em pedículos para transposição através do anel orbital. Para fixação da gordura utiliza-se fio de polipropileno 6.0 passado através da pele. Três a quatro pontos mantém o pedículo na posição durante a fase de cicatrização podendo ser retirados após 3 a 5 dias.

Enxerto de gordura também tem sido usado para preencher a deformidade do sulco lacrimal. ${ }^{12}$ Nessa técnica utiliza-se gordura da porção lateral da coxa por ser mais avascular e de natureza não fibrótica, ${ }^{12}$ aspirando-se em seringa com baixa pressão negativa. Após feita a decantação por 15 a 60 minutos ou centrifugação em seringas de $10 \mathrm{ml}$ por 10 a 60 segundos em $3600 \mathrm{rpm}$, injeta-se, com seringas de $1 \mathrm{ml}$ em região subcutânea, volume suficiente para causar hipercorreção de até $30 \%$ no sulco lacrimal.

Até mesmo implantes aloplásticos tem sido usado para corrigir o sulco. Flowers ${ }^{2}$ desenvolveu um implante malar suborbital em quatro formatos e três espessuras diferentes. Propôs a inserção do mesmo através de cinco técnicas possíveis: via incisão de blefaroplastia, transoral via incisão adjuvante de fossa canina, transconjuntival, incisão direta no sulco lacrimal e via procedimentos de facelift. Apesar dos riscos, o procedimento geralmente promove a correção dessa deformidade da região palpebral inferior.

Vários trabalhos têm sido publicados enfatizando o uso de preenchimento com ácido hialurônico para tratamento do sulco nasojugal..$^{10,13-15}$ Devido a facilidade do uso, previsibilidade dos resultados e ser um procedimento minimamente invasivo, tem ganhado cada vez mais espaço no tratamento dessa deformidade. Várias técnicas tem sido descritas para uso do ácido hialurônico. ${ }^{15}$ Como consenso, recomenda-se o uso do preenchedor em um nível supraperiostal, através ou inferiormente ao anel orbitário, sobre o defeito. A maior parte do preenchedor deve situar-se logo abaixo do músculo orbicular. São recomendados volumes seriados de 0,02 a $0,05 \mathrm{ml}$ por ponto de injeção perpendicular acima do osso cerca de 2 a $3 \mathrm{~mm}$ entre si. O volume injetado dependerá da severidade da depressão. ${ }^{15}$

\section{CONCLUSÃO}

A deformidade do sulco nasojugal apresenta uma ampla gama de tratamentos possíveis, o que, de certa forma, denota a inexistência de uma intervenção ideal. Vários artigos publicados demonstram técnicas mais invasivas em combinação com outras cirurgias, enquanto em outros, técnicas minimamente invasivas como o preenchimento do sulco com gordura ou ácido hialurônico são utilizados. O cirurgião deve ter inicialmente o domínio da anatomia periorbital e conhecimento da técnica utilizada para tratamento adequado do sulco nasojugal. 


\section{REFERÊNCIAS}

1. Espinoza GM, Holds JB. Evaluation and treatment of the tear trough deformity in lower blepharoplasty. Semin Plast Surg. 2007;21(1):57-64.

2. Flowers RS. Tear trough implants for correction of tear trough deformity. Clin Plast Surg. 1993;20(2):403-15.

3. Loeb R. Fat pad sliding and fat grafting for leveling lid depressions. Clin Plast Surg. 1981;8(4):757-76.

4. Steinsapir KD. Aesthetic and restorative midface lifting with handcarved, expanded polytetrafluoroethylene orbital rim implants. Plast Reconstr Surg. 2003;111(5):1727-37.

5. Trepsat F. Periorbital rejuvenation combining fat grafting and blepharoplasties. Aesthetic Plast Surg. 2003;27(4):243-53.

6. Sasaki GH, Cohen AT. Meloplication of the malar fat pads by percutaneous cable-suture technique for midface rejuvenation: outcome study. Plast Reconstr Surg. 2002;110(2):635-54.

7. Lee S, Isse N. Barbed polypropylene sutures for midface elevation: early results. Arch Facial Plast Surg. 2005;7(1):55-61.

8. Moelleken BR. The superficial subciliary cheek lift: a technique for rejuvenating the infraorbital region and nasojugal groove: a review of 71 patients. Plast Reconstr Surg. 1999;104(4):1863-74.
9. Hamra ST. Arcus marginalis release and orbital fat preservation in midface rejuvenation. Plast Reconstr Surg. 1995;96(2):354-62.

10. Kane MA. Treatment of tear trough deformity and lower lid bowing with injectable hyaluronic acid. Aesthetic Plast Surg. 2005;29(5):363-7.

11. Goldberg RA. Transconjunctival orbital fat repositioning: transposition of orbital fat pedicles into a subperiosteal pocket. Plast Reconstr Surg. 2000;105(2):743-51.

12. Butterwick KJ, Nootheti PK, Hsu JW, Goldman MP. Autologous fat transfer: an in-depth look at varying concepts and techniques. Facial Plast Surg Clin North Am. 2007;15:99-111.

13. De Pasquale A, Russa G, Pulvirenti M, Di Rosa L. Hyaluronic acid filler injections for tear-trough deformity: injection technique and high-frequency ultrasound follow-up evaluation. Aesthetic Plast Surg. 2013;37(3):587-91.

14. Morley AM, Malhotra R. Use of hyaluronic acid filler for teartrough rejuvenation as an alternative to lower eyelid surgery. Ophthal Plast Reconstr Surg. 2011;27(2):69-73.

15. Sharad J. Dermal fillers for the treatment of tear trough deformity: a review of anatomy, treatment techniques, and their outcomes. J Cutan Aesthet Surg. 2012:5(4)229-38.

\section{Como citar:}

Machado WA, Pessoa SG, Muniz VV. Análise crítica das opções terapêuticas para deformidades do sulco lacrimal: revisão da literatura. Rev Med UFC. 2017 jan-abr;57(1):43-47. 